\title{
Utilizing Extracted Fungal Pigments for Wood Spalting: A Comparison of Induced Fungal Pigmentation to Fungal Dyeing
}

\author{
Sara C. Robinson, Genevieve Weber, Eric Hinsch, \\ Sarath M. Vega Gutierrez, Lauren Pittis, and Shawn Freitas \\ Department of Wood Science and Engineering, 119 Richardson Hall, Oregon State University, Corvallis, OR 97331, USA \\ Correspondence should be addressed to Sara C. Robinson; sara.robinson@oregonstate.edu
}

Received 28 May 2014; Revised 24 September 2014; Accepted 24 September 2014; Published 7 October 2014

Academic Editor: Mangala Singh

Copyright (C) 2014 Sara C. Robinson et al. This is an open access article distributed under the Creative Commons Attribution License, which permits unrestricted use, distribution, and reproduction in any medium, provided the original work is properly cited.

\begin{abstract}
The lengthy time periods required by current spalting methods prohibit the economically viable commercialization of spalted wood on a large scale. This work aimed to compare the effects of induced spalting in 16 Pacific Northwest woods using three common spalting fungi, Chlorociboria aeruginosa, Scytalidium cuboideum, and Scytalidium ganodermophthorum, with the significantly less time-consuming treatment of these woods using dichloromethane-extracted green, red, and yellow pigments from the same fungi. For pigment extracts, the dosage required for a pigment to internally color various wood species to $30 \%$ internal coverage was investigated. With few exceptions, treatment with pigment extracts outperformed induced spalting in terms of percent internal color coverage. Cottonwood consistently performed best with all three pigment solutions, although chinkapin performed as well as cottonwood with the red pigment, and Port Orford cedar performed as well with the yellow pigment. While no wood species showed $30 \%$ internal color coverage with the green pigment solution, a number of additional species, including pacific silver fir, madrone, dogwood, and mountain hemlock showed internal color coverage on the order of $20-30 \%$ for red and/or yellow. Cottonwood was determined to be the best suited wood species for this type of spalting application.
\end{abstract}

\section{Introduction}

Spalting refers to color changes in wood as a result of penetrating fungal colonization. Spalted wood has a long history of use in woodcraft and art, dating back at least as far as the 15th century [1], and attempts at inducing spalting in clear wood have been under scientific investigation since the mid-1800s [2].

Spalting is of particular value to the woodworking community due to the unique visual nature of the wood and is of growing interest to companies who seek a method of mass production so that spalted wood can be made readily available to those who utilize it. Unfortunately, although substantial early research was conducted on the pigmenting ascomycetes responsible for spalting [2-9], late 20th and early 21st century spalting research has focused almost entirely on the induction of melanized zone line-type spalting, with little regard to the more brightly colored fungal pigments [10].

Recent investigations into the induced spalting of wood, whether to achieve zone lines or pigmentation, has relied entirely on live fungal culture inoculations. In these methods, either small pieces of sterilized wood [11] or larger pieces of freshly cut wood [12] are inoculated with actively growing malt agar cultures of a select group of fungi, incubated under controlled heat and humidity conditions, then dried, and tested after an average cycle of 12 weeks to determine if internal spalting has occurred. Although the 12-week standard time period is substantially shorter and more controllable than simply leaving freshly cut wood on the forest floor to spalt naturally, it is still too long of a time frame to make 
induced spalting economically viable for commercialization on a large scale.

A new method of spalting was recently investigated, where fungi grown on malt agar plates amended with sterile, finely ground white rotted maple [13] were extracted with various organic solvents in an effort to collect the pigment [14]. Dichloromethane was found to be the most effective solvent. Pressure treating of wood samples with this extracted pigment proved to be very successful for surface pigmentation but did not significantly produce any visible colors internally [15]. However the application was very fast, taking an hour to fully color the outside of the test samples.

Alternative uses for the extracted pigments have also been investigated, namely, their potential use as fabric dyes [16]. The most effective method for coloring the test fiber strips was found to be measured droplet application, which completely penetrated and colored the fabric samples.

In an effort to find a method of application for the extracted pigments that generates internal wood coloration of at least $30 \%$ coverage (a standard set in previous laboratory spalting research [10]), the goal of this research was to test the drop dosage required for a pigment solution carried in dichloromethane (DCM) to internally color various wood species, using pigments extracted from the spalting fungi Scytalidium cuboideum (Sacc. and Ellis) Sigler and Kang (red/pink pigment), Scytalidium ganodermophthorum Kang, Sigler, Y.W., Lee, and S.H., Yun (yellow pigment), and Chlorociboria aeruginosa (Oeder) Seaver (blue-green pigment called "xylindein"). The results of this study will be of particular value to industries looking for fast, repeatable methods for spalting wood without the direct use of live fungal cultures or incubators.

\section{Materials and Methods}

Three fungi were selected for use due to the prolific amount of extracellular pigment produced in culture and their history of use as spalting fungi: S. cuboideum UAMH 4802 (isolated from treated red oak lumber, location unknown), S. ganodermophthorum UAMH 10320 (isolated from oak wood logs in Gyeonggi Province, South Korea), and C. aeruginosa UAMH 11657 (isolated from a decaying hardwood log in Haliburton, ON, Canada). Cultures were grown as described in Robinson [13] on malt agar plates amended with white-rotted maple, and pigments were extracted and solubilized in DCM as described in Robinson et al. [14].

A wide selection of wood species was tested: ash (Fraxinus latifolia Benth.), dogwood (Cornus nuttallii Audubon), chinkapin (Chrysolepis chrysophylla Douglas ex Hook.), cottonwood (Populus trichocarpa Torr. and A. Gray), Douglas fir (Pseudotsuga menziesii (Mirb.)), lodgepole pine (Pinus contorta Dougl. ex. Loud), madrone (Arbutus menziesii Pursh), mountain hemlock (Tsuga mertensiana (Bong.)), myrtle (Umbellularia californica (Hook. and Arn.)), noble fir (Abies procera Rehd.), Oregon maple (Acer macrophyllum Pursh), pacific silver fir (Abies amabilis (Douglas ex Loudon)), Port Orford cedar (Chamaecyparis lawsoniana (A. Murray)), red alder (Alnus rubra Bong.), redwood (Sequoia sempervirens
(Lamb. ex. Don)), sweet cherry (Prunus avium (L.)), and sugar maple (Acer saccharum Marsh.).

As a comparison, the fungi were first inoculated onto the wood blocks per the standard protocol described in Robinson et al. [17], with nine replicates per wood species per fungus. Following the eight week incubation in vermiculite jars the blocks were dried, cut in half to expose a radial plane, scanned at 3200 dpi on an Epson Perfection V370 Photo Scanner, and analyzed for percent internal area coverage of color using ImageJ and the Threshold Color plugin, following the protocol developed in Robinson et al. [18].

Solubilized pigments were standardized based upon their color (CIE $L^{*} a^{*} b^{*}$ values) using a Konica Minolta CR-5 chroma meter. Color uniformity was sought for extracts utilized in testing. Standards used were \pm 2.0 from $L^{*} a^{*} b^{*}$ values from $C$. aeruginosa $L^{*}=82.28, a^{*}=-11.06, b^{*}=$ -5.40 ; S. cuboideum $L^{*}=82.32, a^{*}=26.84, b^{*}=13.19$; and S. ganodermophthorum $L^{*}=95.46, a^{*}=-3.00, b^{*}=$ 8.15. This method of standardization was utilized instead of concentration by dry weight as the chromatic difference in pigment produced by these fungi can vary between plates, even within the same strain. For results that could be properly analyzed it was important that $L^{*} a^{*} b^{*}$ values match as closely as possible. A rough conversion for the $L^{*} a^{*} b^{*}$ values of $S$. cuboideum and $C$. aeruginosa to dry pigment weight can be found in Robinson et al. [14]. Uniformity was achieved by concentrating or diluting extracts until the $L^{*} a^{*} b^{*}$ values fell within the acceptable range.

To best mimic the likely conditions under which the solubilized pigments would be utilized under commercial settings, wood samples were kiln dried to $8 \%$ moisture content (MC) then allowed to equilibrate to $10 \% \mathrm{MC}$ with ambient indoor air. The samples were cut into $14 \mathrm{~mm}$ cubes so comparisons could be made in terms of pigment penetration depth between inoculated samples and pigmented samples.

Solubilized pigments were applied with a 5.75 inch disposable borosilicate glass pipet (VWR, Radnor PA), with the average volume of solution per drop from the pipet at $0.0165 \mathrm{~mL}$. Blocks were laid out $1 \mathrm{~cm}$ apart, transverse side up, on glass sheets, under a fume hood. Drops were placed at a variable rate depending on wood species, species with smaller vessels/tracheids, and higher extractive contents had drops placed more slowly, so that the solution did not run down the side of the block. Nine separate levels of solution were applied: $1,5,10,20,40$, and 60 drops of straight solution; 10 drops of unaltered DCM, pausing for ten minute, then applying 10 drops of pigment; applying 28 drops of solution, waiting 24 hours and then applying another 28 drops; applying 50 drops of solution, waiting 24 hours and then applying another 50 drops.

After treatment all blocks were allowed to air-dry for 48 hours. They were then cut in half longitudinally, scanned, and analyzed for percent color coverage as described above.

2.1. Data Analysis. Data were separated by fungus species. A two-way ANOVA followed by Tukey's HSD was performed with wood species and test type as the independent variables and internal color coverage as the dependent variable. All statistics were performed on SAS version 9.3. 


\section{Results}

3.1. S. cuboideum. The two-way ANOVA for S. cuboideum was significant at $P<0.0001$. Wood species, test type, and the interaction of wood and test type were all significant at $P<0.0001$. Results are summarized in Table 1. Overall, cottonwood and chinkapin performed the best in terms of internal color coverage, with 60 drops on cottonwood and the 50 pause 50 drops having significantly more internal color coverage than any other test and wood species with the exception of chinkapin at 60 drops, chinkapin at 50 pause 50 , and pacific silver fir at 50 pause 50 .

Cherry and myrtle did not perform as well with the drip testing as they did with direct inoculation in terms of internal color coverage, although the difference was only significant in myrtle. Ash, Douglas fir, lodgepole pine, red alder, redwood, and sugar maple exhibited no significant differences in internal color coverage regardless of treatment. The most effective number of pigment drops varied by wood species; chinkapin and cottonwood showed the most internal color at 60 and 50 pause 50 drops, dogwood, madrone, pacific silver fir and mountain hemlock at 50 pause 50 , noble fir at 60 drops (although this amount was only significantly different from 1,5 , and DCM followed by 10 drops), and Oregon maple and Port Orford cedar at 60 drops.

Only chinkapin, cottonwood, and pacific silver fir showed at least $30 \%$ internal color coverage, although dogwood, madrone, and myrtle had coverage in the midtwenties.

3.2. C. aeruginosa. The two-way ANOVA for C. aeruginosa was significant at $P<0.0001$. Wood species, test type, and the interaction of wood and test type were all significant at $P<$ 0.0001 . Cottonwood at 60 drops showed the most internal color coverage, although the amount was not significantly different from cottonwood at 50 pause 50, Port Orford cedar at 60 drops, or chinkapin at 60 drops. Unsurprisingly, none of the inoculated samples showed significant internal color. Ash, cherry, dogwood, Douglas fir, lodgepole pine, and myrtle showed no significant internal color coverage regardless of number of drops applied.

None of the test wood species showed over $30 \%$ internal color coverage regardless of test type. However, in every wood species where internal green pigment occurred there was greater coverage from the drop testing than the inoculation.

3.3. S. ganodermophthorum. The two-way ANOVA for $S$. ganodermophthorum was significant at $P<0.0001$. Wood species, test type, and the interaction of wood and test type were all significant at $P<0.0001$. The yellow pigment only worked well on a few species. Cottonwood at 60 drops showed the most internal color coverage, although it was not significantly different from pacific silver fir at 50 pause 50 , cottonwood at 50 pause 50 , and Port Orford cedar at 50 pause 50 or 60 drops. Ash, cherry, chinkapin, dogwood, Douglas fir, lodgepole pine, madrone, mountain hemlock, myrtle, noble fir, Oregon maple, red alder, redwood, and sugar maple showed no significant internal color coverage regardless of test. The most effective number of pigment drops was 60 for cottonwood, 50 pause 50 for pacific silver fir, and 60 and 50 pause 50 for Port Orford cedar.

Only chinkapin and cottonwood showed at least 30\% internal color, although mountain hemlock, pacific silver fir, and Port Orford cedar showed at least 20\% internal coverage.

By 60 drops, the yellow pigment solution was able to completely travel the transverse length of all the wood species and come out the opposite side. The first occurrence of the solution moving completely through the test blocks differed between wood species and pigment. In chinkapin and cottonwood with S.c , five drops of the red solution were enough to move completely through the wood. The green pigment was able to move completely through cottonwood, pacific silver fir, chinkapin, ash, and sugar maple with the pretreatment of DCM followed by 10 drops of solution. The yellow pigment did not begin to show on the opposite transverse side of the blocks until dosage hit 20 drops, and even then the color only came through on cottonwood and chinkapin.

\section{Discussion}

Although nearly all wood species were beginning to show internal pigmentation by 60 or 50 pause 50 drops, the testing was not continued to higher levels due to the excessive runoff of the solution through the opposite transverse face of the blocks.

A plausible reason for why DCM is able to run through the blocks so rapidly is its diffusion through the cell wall material. Liquids can move through wood by two main mechanisms: capillary action and diffusion. Capillary action takes advantage of the cell lumen space and pores, while diffusion takes advantage of the cell wall material [19]. Wood anatomy can significantly affect capillary action and it is fairly easy to clog lumens and pores once flow has been established. Diffusion on the other hand is driven primarily by chemical potential and is not stopped by anatomical changes [20]. Compared to water, DCM has considerable chemical potential when travelling through wood as it has an extremely high vapor pressure, low boiling point, and major concentration gradient and is a polar aprotic solvent less likely to interact with cellulose than a polar protic solvent like water [21].

Of the wood species tested, cottonwood performed the best with all three fungal species. It is of special interest to note that cottonwood was the only wood species to have significant internal color coverage with all three pigments at 60 drops and 50 pause 50 . This is likely due to the low extractive content and high porosity of cottonwood. This wood species is likely the best suited for this type of spalting application.

Chinkapin also did well with $S$. cuboideum and $C$. aeruginosa, while Port Orford cedar did well with all three fungi, but did not show nearly as much internal color as cottonwood. Anatomically these three wood species are very different; chinkapin and cottonwood are both hardwoods and have unicellular rays; however, chinkapin is ring porous while cottonwood is diffuse porous [22]. Chinkapin also has a moderate level of tyloses, while cottonwood has none. Port Orford cedar is a conifer with a gradual earlywood 
TABLE 1: Mean percent internal spalting from extracted fungal pigments. Different letters represent statistically significant differences at alpha $=0.05$ within each color (red, green, or yellow) .

\begin{tabular}{|c|c|c|c|c|}
\hline Wood & Test & Mean \% internal spalt red & Mean \% internal spalt green & Mean \% internal spalt yellow \\
\hline \multirow{9}{*}{ Ash } & Inoculation & $0(\mathrm{~L})$ & $0(\mathrm{~F})$ & $0.4(\mathrm{OPQ})$ \\
\hline & 1 drop & $0(\mathrm{~L})$ & $0(\mathrm{~F})$ & $0(\mathrm{Q})$ \\
\hline & 5 drops & $0(\mathrm{~L})$ & $0(\mathrm{~F})$ & $0(\mathrm{Q})$ \\
\hline & 10 drops & $0.1(\mathrm{~L})$ & $0(\mathrm{~F})$ & 0.8 (MNOPQ) \\
\hline & $\mathrm{dcm}+10$ & $0(\mathrm{~L})$ & $0(\mathrm{~F})$ & $0(\mathrm{Q})$ \\
\hline & 28 pause 28 & $1.0(\mathrm{KL})$ & $0(\mathrm{~F})$ & 4.7 (IJKLMNOPQ) \\
\hline & 40 drops & $0.3(\mathrm{KL})$ & $0(\mathrm{~F})$ & 2.7 (JKLMNOPQ) \\
\hline & 50 pause 50 & 3.0 (IJKL) & $0(\mathrm{~F})$ & 14.7 (EFGHIJKLMNO) \\
\hline & 60 drops & 4.3 (HIJKL) & $0(\mathrm{~F})$ & 5.7 (IJKLMNOPQ) \\
\hline \multirow{9}{*}{ Cherry } & Inoculation & 18.1 (DEFGHIJ) & $0(\mathrm{~F})$ & $0(\mathrm{Q})$ \\
\hline & 1 drop & $0(\mathrm{~L})$ & $0(\mathrm{~F})$ & $0(\mathrm{Q})$ \\
\hline & 5 drops & $0(\mathrm{~L})$ & $0(\mathrm{~F})$ & $0(\mathrm{Q})$ \\
\hline & 10 drops & $0(\mathrm{~L})$ & $0(\mathrm{~F})$ & $0(\mathrm{Q})$ \\
\hline & $\mathrm{dcm}+10$ & $0(\mathrm{~L})$ & $0(\mathrm{~F})$ & $0(\mathrm{Q})$ \\
\hline & 28 pause 28 & $0(\mathrm{~L})$ & $0(\mathrm{~F})$ & $0.3(\mathrm{PQ})$ \\
\hline & 40 drops & $0(\mathrm{~L})$ & $0(\mathrm{~F})$ & $0.3(\mathrm{PQ})$ \\
\hline & 50 pause 50 & $2.3(\mathrm{IJKL})$ & $0(\mathrm{~F})$ & 3.0 (JKLMNOPQ) \\
\hline & 60 drops & 2.7 (IJKL) & $0(\mathrm{~F})$ & 4.0 (JKLMNOPQ) \\
\hline \multirow{9}{*}{ Chinkapin } & Inoculation & 3.3 (HIJKL) & $0(\mathrm{~F})$ & 4.0 (JKLMNOPQ) \\
\hline & 1 drop & $0(\mathrm{~L})$ & $0(\mathrm{~F})$ & $0(\mathrm{Q})$ \\
\hline & 5 drops & $0(\mathrm{~L})$ & $0(\mathrm{~F})$ & $0.3(\mathrm{PQ})$ \\
\hline & 10 drops & 1.7 (JKL) & $0(\mathrm{~F})$ & 1.0 (MNOPQ) \\
\hline & $\mathrm{dcm}+10$ & 4.5 (HIJKL) & $0(\mathrm{~F})$ & 2.2 (JKLMNOPQ) \\
\hline & 28 pause 28 & 2.7 (IJKL) & $0(\mathrm{~F})$ & 13.8 (FGHIJKLMNOPQ) \\
\hline & 40 drops & $1.0(\mathrm{KL})$ & $0(\mathrm{~F})$ & 5.7 (IJKLMNOPQ) \\
\hline & 50 pause 50 & $48.5(\mathrm{~B})$ & $0(\mathrm{~F})$ & 12.3 (GHIJKLMNOPQ) \\
\hline & 60 drops & $49.1(\mathrm{~B})$ & $0(\mathrm{~F})$ & $33.0(\mathrm{ABCD})$ \\
\hline \multirow{9}{*}{ Cottonwood } & Inoculation & 6.1 (HIJKL) & 7.8 (BCDEF) & 0.4 (OPQ) \\
\hline & 1 drop & $0(\mathrm{~L})$ & $0(\mathrm{~F})$ & 1.0 (MNOPQ) \\
\hline & 5 drops & 3.3 (HIJKL) & $0(\mathrm{~F})$ & $0(\mathrm{Q})$ \\
\hline & 10 drops & 9.1 (EFGHIJKL) & $0(\mathrm{~F})$ & 1.7 (LMNOPQ) \\
\hline & $\mathrm{dcm}+10$ & 4.5 (HIJKL) & $0(\mathrm{~F})$ & 1.0 (MNOPQ) \\
\hline & 28 pause 28 & 5.3 (HIJKL) & $0(\mathrm{~F})$ & 11.2 (GHIJKLMNOPQ) \\
\hline & 40 drops & 3.9 (HIJKL) & $0(\mathrm{~F})$ & 6.3 (HIJKLMNOPQ) \\
\hline & 50 pause 50 & $50.3(\mathrm{AB})$ & $11.7(\mathrm{ABC})$ & $41.3(\mathrm{AB})$ \\
\hline & 60 drops & $66.1(\mathrm{~A})$ & $16.7(\mathrm{~A})$ & $44.3(\mathrm{~A})$ \\
\hline \multirow{9}{*}{ Dogwood } & Inoculation & $0.3(\mathrm{KL})$ & $0(\mathrm{~F})$ & $0(\mathrm{Q})$ \\
\hline & 1 drop & $0(\mathrm{~L})$ & $0(\mathrm{~F})$ & $0(\mathrm{Q})$ \\
\hline & 5 drops & $0(\mathrm{~L})$ & $0(\mathrm{~F})$ & $0(\mathrm{Q})$ \\
\hline & 10 drops & $0(\mathrm{~L})$ & $0(\mathrm{~F})$ & $0.2(\mathrm{Q})$ \\
\hline & $\mathrm{dcm}+10$ & $0(\mathrm{~L})$ & $0(\mathrm{~F})$ & $0(\mathrm{Q})$ \\
\hline & 28 pause 28 & $1.0(\mathrm{KL})$ & $0(\mathrm{~F})$ & 8.2 (HIJKLMNOPQ) \\
\hline & 40 drops & $0.8(\mathrm{KL})$ & $0(\mathrm{~F})$ & 1.4 (LMNOPQ) \\
\hline & 50 pause 50 & 24.0 (DEFG) & $0(\mathrm{~F})$ & 2.0 (LMNOPQ) \\
\hline & 60 drops & 10.7 (EFGHIJKL) & $0(\mathrm{~F})$ & 10.0 (GHIJKLMNOPQ) \\
\hline \multirow{6}{*}{ Douglas fir } & Inoculation & $0.4(\mathrm{KL})$ & $0(\mathrm{~F})$ & 12.7 (FGHIJKLMNOPQ) \\
\hline & 1 drop & $0(\mathrm{~L})$ & $0(\mathrm{~F})$ & $0(\mathrm{Q})$ \\
\hline & 5 drops & $0(\mathrm{~L})$ & $0(\mathrm{~F})$ & $0(\mathrm{Q})$ \\
\hline & 10 drops & $0(\mathrm{~L})$ & $0(\mathrm{~F})$ & $0.3(\mathrm{PQ})$ \\
\hline & $\mathrm{dcm}+10$ & $0(\mathrm{~L})$ & $0(\mathrm{~F})$ & $0(\mathrm{Q})$ \\
\hline & 28 pause 28 & $0.8(\mathrm{KL})$ & $0(\mathrm{~F})$ & 7.5 (HIJKLMNOPQ) \\
\hline
\end{tabular}


TABle 1: Continued.

\begin{tabular}{|c|c|c|c|c|}
\hline Wood & Test & Mean \% internal spalt red & Mean \% internal spalt green & Mean \% internal spalt yellow \\
\hline & 40 drops & $1.9(\mathrm{JKL})$ & $0(\mathrm{~F})$ & 3.1 (JKLMNOPQ) \\
\hline & 50 pause 50 & 12.3 (EFGHIJKL) & $0(\mathrm{~F})$ & 13.3 (FGHIJKLMNOPQ) \\
\hline & 60 drops & 14.7 (EFGHIJKL) & $0(\mathrm{~F})$ & 8.3 (HIJKLMNOPQ) \\
\hline \multirow{9}{*}{ Lodgepole pine } & Inoculation & $1.0(\mathrm{KL})$ & $0(\mathrm{~F})$ & $0.1(\mathrm{Q})$ \\
\hline & 1 drop & $0(\mathrm{~L})$ & $0(\mathrm{~F})$ & $0(\mathrm{Q})$ \\
\hline & 5 drops & $0(\mathrm{~L})$ & $0(\mathrm{~F})$ & $0(\mathrm{Q})$ \\
\hline & 10 drops & $0.2(\mathrm{KL})$ & $0(\mathrm{~F})$ & $0.2(\mathrm{Q})$ \\
\hline & $\mathrm{dcm}+10$ & $0(\mathrm{~L})$ & $0(\mathrm{~F})$ & $0(\mathrm{Q})$ \\
\hline & 28 pause 28 & 3.8 (HIJKL) & $0(\mathrm{~F})$ & 3.5 (JKLMNOPQ) \\
\hline & 40 drops & 3.1 (HIJKL) & $0(\mathrm{~F})$ & 2.9 (JKLMNOPQ) \\
\hline & 50 pause 50 & 8.3 (FGHIJKL) & $1.0(\mathrm{EF})$ & 9.3 (HIJKLMNOPQ) \\
\hline & 60 drops & 7.6 (GHIJKL) & $0(\mathrm{~F})$ & 8.0 (HIJKLMNOPQ) \\
\hline \multirow{9}{*}{ Madrone } & Inoculation & $0.6(\mathrm{KL})$ & $0(\mathrm{~F})$ & $0(\mathrm{Q})$ \\
\hline & 1 drop & $0(\mathrm{~L})$ & $0(\mathrm{~F})$ & $0(\mathrm{Q})$ \\
\hline & 5 drops & $0(\mathrm{~L})$ & $0(\mathrm{~F})$ & $0(\mathrm{Q})$ \\
\hline & 10 drops & $0(\mathrm{~L})$ & $0(\mathrm{~F})$ & $0(\mathrm{Q})$ \\
\hline & $\mathrm{dcm}+10$ & $0(\mathrm{~L})$ & $0(\mathrm{~F})$ & $0(\mathrm{Q})$ \\
\hline & 28 pause 28 & $0(\mathrm{~L})$ & $0(\mathrm{~F})$ & 0.6 (NOPQ) \\
\hline & 40 drops & $0(\mathrm{~L})$ & $0(\mathrm{~F})$ & 2.2 (JKLMNOPQ) \\
\hline & 50 pause 50 & $25.0(\mathrm{DE})$ & $0(\mathrm{~F})$ & 3.7 (JKLMNOPQ) \\
\hline & 60 drops & $0(\mathrm{~L})$ & $0(\mathrm{~F})$ & $0(\mathrm{Q})$ \\
\hline \multirow{9}{*}{ Mountain hemlock } & Inoculation & $0(\mathrm{~L})$ & $0(\mathrm{~F})$ & 0.8 (MNOPQ) \\
\hline & 1 drop & $0(\mathrm{~L})$ & $0(\mathrm{~F})$ & $0(\mathrm{Q})$ \\
\hline & 5 drops & $0(\mathrm{~L})$ & $0(\mathrm{~F})$ & $0(\mathrm{Q})$ \\
\hline & 10 drops & $1.9(\mathrm{JKL})$ & $0(\mathrm{~F})$ & $0(\mathrm{Q})$ \\
\hline & $\mathrm{dcm}+10$ & $0(\mathrm{~L})$ & $0(\mathrm{~F})$ & $0(\mathrm{Q})$ \\
\hline & 28 pause 28 & 2.5 (IJKL) & $0(\mathrm{~F})$ & 18.5 (EFGHI) \\
\hline & 40 drops & 3.5 (HIJKL) & $0(\mathrm{~F})$ & 14.8 (EFGHIJKLMN) \\
\hline & 50 pause 50 & $31.7(\mathrm{CD})$ & 7.3 (BCDEF) & 26.7 (CDEF) \\
\hline & 60 drops & 15.3 (DEFGHIJKL) & $0(\mathrm{~F})$ & 3.3 (JKLMNOPQ) \\
\hline \multirow{9}{*}{ Myrtle } & Inoculation & 24.3 (DEF) & $0(\mathrm{~F})$ & $0(\mathrm{Q})$ \\
\hline & 1 drop & $0(\mathrm{~L})$ & $0(\mathrm{~F})$ & $0(\mathrm{Q})$ \\
\hline & 5 drops & $0(\mathrm{~L})$ & $0(\mathrm{~F})$ & $0(\mathrm{Q})$ \\
\hline & 10 drops & $0(\mathrm{~L})$ & $0(\mathrm{~F})$ & $0(\mathrm{Q})$ \\
\hline & $\mathrm{dcm}+10$ & $0(\mathrm{~L})$ & $0(\mathrm{~F})$ & $0(\mathrm{Q})$ \\
\hline & 28 pause 28 & $0(\mathrm{~L})$ & $0(\mathrm{~F})$ & 0.6 (NOPQ) \\
\hline & 40 drops & $0(\mathrm{~L})$ & $0(\mathrm{~F})$ & $0(\mathrm{Q})$ \\
\hline & 50 pause 50 & 2.3 (IJKL) & $0(\mathrm{~F})$ & 1.3 (LMNOPQ) \\
\hline & 60 drops & 4.1 (HIJKL) & $0(\mathrm{~F})$ & $0(\mathrm{Q})$ \\
\hline \multirow{9}{*}{ Moble fir } & Inoculation & $0.2(\mathrm{KL})$ & $0(\mathrm{~F})$ & 1.8 (LMNOPQ) \\
\hline & 1 drop & $0(\mathrm{~L})$ & $0(\mathrm{~F})$ & $0(\mathrm{Q})$ \\
\hline & 5 drops & $0(\mathrm{~L})$ & $0(\mathrm{~F})$ & $0(\mathrm{Q})$ \\
\hline & 10 drops & $0.8(\mathrm{KL})$ & $0(\mathrm{~F})$ & $0(\mathrm{Q})$ \\
\hline & $\mathrm{dcm}+10$ & $0(\mathrm{~L})$ & $0(\mathrm{~F})$ & $0(\mathrm{Q})$ \\
\hline & 28 pause 28 & $1.7(\mathrm{JKL})$ & 6.7 (BCDEF) & 8.7 (HIJKLMNOPQ) \\
\hline & 40 drops & 4.3 (HIJKL) & $0(\mathrm{~F})$ & 12.6 (FGHIJKLMNOPQ) \\
\hline & 50 pause 50 & 16.3 (DEFGHIJKL) & $0(\mathrm{~F})$ & 18.3 (EFGHI) \\
\hline & 60 drops & 16.7 (DEFGHIJK) & $0(\mathrm{~F})$ & 16.3 (EFGHIJ) \\
\hline
\end{tabular}


TABle 1: Continued.

\begin{tabular}{|c|c|c|c|c|}
\hline Wood & Test & Mean \% internal spalt red & Mean \% internal spalt green & Mean \% internal spalt yellow \\
\hline \multirow{9}{*}{ Oregon maple } & Inoculation & 3.1 (HIJKL) & $0.3(\mathrm{~F})$ & 9.8 (HIJKLMNOPQ) \\
\hline & 1 drop & $0(\mathrm{~L})$ & $1.7(\mathrm{DEF})$ & $0(\mathrm{Q})$ \\
\hline & 5 drops & $0(\mathrm{~L})$ & $2.0(\mathrm{DEF})$ & $0(\mathrm{Q})$ \\
\hline & 10 drops & $0(\mathrm{~L})$ & $0(\mathrm{~F})$ & 0.7 (NOPQ) \\
\hline & $\mathrm{dcm}+10$ & $0(\mathrm{~L})$ & $0(\mathrm{~F})$ & $0(\mathrm{Q})$ \\
\hline & 28 pause 28 & $0.5(\mathrm{KL})$ & $0(\mathrm{~F})$ & 1.8 (LMNOPQ) \\
\hline & 40 drops & $0.3(\mathrm{KL})$ & $0(\mathrm{~F})$ & 1.4 (LMNOPQ) \\
\hline & 50 pause 50 & 2.3 (IJKL) & $0(\mathrm{~F})$ & 5.7 (IJKLMNOPQ) \\
\hline & 60 drops & 18.8 (DEFGHI) & $0(\mathrm{~F})$ & 15.7 (EFGHIJK) \\
\hline \multirow{9}{*}{ Pacific silver fir } & Inoculation & $0(\mathrm{~L})$ & $0(\mathrm{~F})$ & $0.2(\mathrm{Q})$ \\
\hline & 1 drop & $0(\mathrm{~L})$ & $0(\mathrm{~F})$ & $0(\mathrm{Q})$ \\
\hline & 5 drops & $1.4(\mathrm{KL})$ & $0(\mathrm{~F})$ & $0(\mathrm{Q})$ \\
\hline & 10 drops & $1.6(\mathrm{JKL})$ & $0(\mathrm{~F})$ & 2.0 (LMNOPQ) \\
\hline & $\mathrm{dcm}+10$ & 3.4 (HIJKL) & $0(\mathrm{~F})$ & 3.2 (JKLMNOPQ) \\
\hline & 28 pause 28 & 2.3 (IJKL) & $0(\mathrm{~F})$ & 15.1 (EFGHIJKLM) \\
\hline & 40 drops & $1.8(\mathrm{JKL})$ & $0(\mathrm{~F})$ & 14.6 (EFGHIJKLMNOP) \\
\hline & 50 pause 50 & $46.7(\mathrm{BC})$ & $14.0(\mathrm{AB})$ & $28.3(\mathrm{BCDE})$ \\
\hline & 60 drops & 19.7 (DEFGH) & 6.7 (BCDEF) & $28.3(\mathrm{BCDE})$ \\
\hline \multirow{9}{*}{ Port Orford cedar } & Inoculation & $1.8(\mathrm{JKL})$ & $0(\mathrm{~F})$ & $0(\mathrm{Q})$ \\
\hline & 1 drop & $0(\mathrm{~L})$ & $0(\mathrm{~F})$ & $0(\mathrm{Q})$ \\
\hline & 5 drops & $1.8(\mathrm{JKL})$ & $0(\mathrm{~F})$ & $0.2(\mathrm{Q})$ \\
\hline & 10 drops & 3.4 (HIJKL) & $0(\mathrm{~F})$ & 1.7 (LMNOPQ) \\
\hline & $\mathrm{dcm}+10$ & $1.0(\mathrm{KL})$ & $0(\mathrm{~F})$ & 1.0 (MNOPQ) \\
\hline & 28 pause 28 & 6.0 (HIJKL) & $0(\mathrm{~F})$ & $24.2(\mathrm{CDEFG})$ \\
\hline & 40 drops & $5.1(\mathrm{HIJKL})$ & $0(\mathrm{~F})$ & $20.1(\mathrm{DEFGH})$ \\
\hline & 50 pause 50 & 19.7 (DEFGH) & $9.7(\mathrm{ABCD})$ & 12.3 (GHIJKLMNOPQ) \\
\hline & 60 drops & $25.4(\mathrm{DE})$ & 8.7 (ABCDE) & $37.7(\mathrm{ABC})$ \\
\hline \multirow{9}{*}{ Red alder } & Inoculation & $0(\mathrm{~L})$ & $0(\mathrm{~F})$ & $0(\mathrm{Q})$ \\
\hline & 1 drop & $0(\mathrm{~L})$ & $0(\mathrm{~F})$ & $0(\mathrm{Q})$ \\
\hline & 5 drops & $0(\mathrm{~L})$ & $0(\mathrm{~F})$ & $0(\mathrm{Q})$ \\
\hline & 10 drops & $0(\mathrm{~L})$ & $0(\mathrm{~F})$ & $0.2(\mathrm{Q})$ \\
\hline & $\mathrm{dcm}+10$ & $0(\mathrm{~L})$ & $0(\mathrm{~F})$ & $0(\mathrm{Q})$ \\
\hline & 28 pause 28 & $0.3(\mathrm{KL})$ & $0(\mathrm{~F})$ & 3.0 (JKLMNOPQ) \\
\hline & 40 drops & $0.7(\mathrm{KL})$ & $0(\mathrm{~F})$ & 3.4 (JKLMNOPQ) \\
\hline & 50 pause 50 & 6.7 (HIJKL) & $0(\mathrm{~F})$ & 3.3 (JKLMNOPQ) \\
\hline & 60 drops & 6.7 (HIJKL) & $1.0(\mathrm{EF})$ & 15.3 (EFGHIJKL) \\
\hline \multirow{9}{*}{ Redwood } & Inoculation & 1.9 (JKL) & $0(\mathrm{~F})$ & $0(\mathrm{Q})$ \\
\hline & 1 drop & $0(\mathrm{~L})$ & $0(\mathrm{~F})$ & $0(\mathrm{Q})$ \\
\hline & 5 drops & $0(\mathrm{~L})$ & $0(\mathrm{~F})$ & $0(\mathrm{Q})$ \\
\hline & 10 drops & $0(\mathrm{~L})$ & $0(\mathrm{~F})$ & $0.3(\mathrm{PQ})$ \\
\hline & $\mathrm{dcm}+10$ & $0(\mathrm{~L})$ & $0(\mathrm{~F})$ & $0(\mathrm{Q})$ \\
\hline & 28 pause 28 & 9.3 (EFGHIJKL) & $0(\mathrm{~F})$ & 3.6 (JKLMNOPQ) \\
\hline & 40 drops & 6.0 (HIJKL) & $0(\mathrm{~F})$ & 3.7 (JKLMNOPQ) \\
\hline & 50 pause 50 & $0(\mathrm{~L})$ & $0(\mathrm{~F})$ & 6.7 (HIJKLMNOPQ) \\
\hline & 60 drops & 9.8 (EFGHIJKL) & $0(\mathrm{~F})$ & 16.3 (EFGHIJ) \\
\hline \multirow{6}{*}{ Sugar maple } & Inoculation & $2.1(\mathrm{JKL})$ & $0(\mathrm{~F})$ & $0.2(\mathrm{Q})$ \\
\hline & 1 drop & $0(\mathrm{~L})$ & $4.7(\mathrm{CDEF})$ & $0(\mathrm{Q})$ \\
\hline & 5 drops & $0(\mathrm{~L})$ & $0.7(\mathrm{EF})$ & $0(\mathrm{Q})$ \\
\hline & 10 drops & $0(\mathrm{~L})$ & $4.3(\mathrm{CDEF})$ & 0.5 (OPQ) \\
\hline & $\mathrm{dcm}+10$ & $0(\mathrm{~L})$ & $0(\mathrm{~F})$ & 0. (PQ) \\
\hline & 28 pause 28 & $0(\mathrm{~L})$ & $0(\mathrm{~F})$ & 8.1 (HIJKLMNOPQ) \\
\hline
\end{tabular}


TABle 1: Continued.

\begin{tabular}{|c|c|c|c|c|}
\hline Wood & Test & Mean \% internal spalt red & Mean \% internal spalt green & Mean \% internal spalt yellow \\
\hline & 40 drops & $2.0(\mathrm{JKL})$ & $0(\mathrm{~F})$ & 6.4 (HIJKLMNOPQ) \\
\hline & 50 pause 50 & $0(\mathrm{~L})$ & $0(\mathrm{~F})$ & $0(\mathrm{Q})$ \\
\hline & 60 drops & 11.3 (EFGHIJKL) & $0(\mathrm{~F})$ & $0(\mathrm{Q})$ \\
\hline
\end{tabular}

to latewood transition. All have average specific gravities in the 0.4 range and none have particularly visually distinct latewood. Anatomically there is little reason why these three species would outperform the others in terms of internal pigmentation.

Assuming that DCM's ability to travel through the specimens so quickly is due to diffusion related to its chemical potential in this matrix and not due to wood anatomy, then further explanation is possible for why certain wood species pigment better than others when utilizing a DCM extract. Diffusion through the cell wall material will bring the DCM extract directly into contact with the components of the cell wall and as a result, the extractive, lignin, and hemicellulose profiles of the woods tested would have a significant effect on both the speed of the diffusion as well as the level of color addition. Potential effects of cell wall composition on the speed of DCM diffusion and the level of color addition are supported by solvent-solute exchange dynamics that are frequently witnessed when performing liquid-liquid extractions, antisolvent crystallizations, and precipitation crystallizations [23-25]. As an organic solvent-solute solution, the DCM extract is not in a fixed state and is actually a changing mix of solute-solvent complexes that are constantly forming and dissociating on picosecond timescales as they struggle to maintain thermal equilibrium conditions. This equilibrium is ultimately composed of varying concentrations of free solute, solute-solvent complexes, and solvent that change based on the conditions the organic solvent-solute solution is exposed to [23].

One of the conditions that can dramatically change the equilibrium is the addition of new solutes to the solvent. When a solvent begins complexing with a new solute that is thermodynamically preferable there will be more association with the new solute and less association with the old. This can either result in more solute-solute reactions and precipitation like that witnessed in salting out or it can result in a solvent exchange similar to liquid-liquid extractions whereby the old solute will preferentially leave the solvent and associate with a more thermodynamically favorable liquid or solid [24, 25]. As a result, in the case of the DCM extract, a wood's particular extractive, lignin, and hemicellulose profile will affect both the number of solutes and the solute balance in the extract, potentially rejecting the dye compounds to the wood matrix in favor of more thermodynamically favorable solutes.

Previous work on extracting spalting fungal pigments has shown that DCM will efficiently remove dye compounds and other extractives from wood and wood/fungal cultures [14]. This means that DCM extracts can form a fairly stable equilibrium with the dye compounds and remain unaffected by the components of the wood cell wall matrix. However, it does not mean that this will be the case with every species of wood and these results suggest that certain wood cell wall compositions are capable of affecting that equilibrium and causing the solvent to deposit the dye compounds in the wood. This also means that extracting dye compounds from wood/fungal cultures composed of these woods may be ineffective or require significantly more solvent to account for the increased level of desirable solutes. While it is also possible to change the balance of solutes by changing the temperature or pressure to swing the thermodynamics in the preferred direction $[24,25]$, in these experiments pressure and temperature were fairly stable, so the most reasonable explanation for the DCM extract to deposit the dye compounds in the wood is solute exchange from the addition of new solutes that changed the equilibrium conditions.

Future work may investigate the different DCM extract profiles of the wood species that dyed well compared to wood species that did not dye well to try and identify wood specific solutes that cause dye compounds to leave the DCM extract. It is also possible that this solute exchange, dye deposition process, is occurring at some level with other wood species as well but is most visible on those with lighter colored wood.

\section{Conclusions}

Cottonwood and chinkapin performed best in terms of internal red pigment coverage, with the most optimal results from the 60 drops and 50 pause 50 drops applications. For the green and yellow pigments, cottonwood again performed the best of the wood species test at the 60 drops application. A number of other wood species showed only slightly less internal pigment than cottonwood and chinkapin and were not significantly different in terms of internal pigment amount. Most notably, Port Orford cedar and cottonwood showed significant amounts of internal color with all three pigments.

\section{Conflict of Interests}

The authors declare that there is no conflict of interests regarding the publication of this paper.

\section{References}

[1] R. A. Blanchette, A. M. Wilmering, and M. Baumeister, “ The use of green-stained wood caused by the fungus Chlorociboria in Intarsia masterpieces from the 15th century," Holzforschung, vol. 46, no. 3, pp. 225-232, 1992.

[2] M. J. Berkeley, Outlines of British Fungology, Lovell Reeve, London, UK, 1860.

[3] C. Liebermann, "Über xylindein," Berichte der Deutschen Chemischen Gesellschaft, vol. 7, no. 2, pp. 1102-1103, 1874. 
[4] R. Hartig, Die Zersetzungserscheinungen des Holzes der Nadelholzbäume und der Eiche in Forstlicher, Botanischer und Chemischer Richtung, Springer, Berlin, Germany, 1878.

[5] E. Eidam, "Blaugrün gefärbtes Holz von Birken und Buchen und blut-bis Cakminroth gefärbtes von Acer negundo," Jahresbericht der Schlesischen Gesellschaft für Vaterländische Cultur, vol. 58, pp. 188-189, 1880.

[6] F. T. Brooks, "Improvements in or relating to colouring and/or preserving wood," English Patent No. 24,595, London, UK, 1913.

[7] H. von Schrenk, "The "bluing" and "red rot" of the western yellow pine, with special reference to the black hills forest reserve," Bureau of Plant Industry-Bulletin no. 36, U.S. Department of Agriculture, 1903.

[8] P. Vuillemin, "Le Bois Verdi," Bulletin de la Societe des Sciences de Nancy Series II, vol. 15, pp. 90-145, 1898.

[9] G. G. Hedgcock, "Studies upon some chromogenic fungi which discolor wood," Missouri Botanical Garden Annual Report 1906:59-114, 1906.

[10] S. C. Robinson, "Developing fungal pigments for "painting" vascular plants," Applied Microbiology and Biotechnology, vol. 93, no. 4, pp. 1389-1394, 2012.

[11] S. C. Robinson, D. L. Richter, and P. E. Laks, "Colonization of sugar maple by spalting fungi," Forest Products Journal, vol. 57, no. 4, pp. 24-32, 2007.

[12] S. C. Robinson, D. Tudor, S. Hipson et al., "Methods of inoculating Acer spp., Populus tremuloides, and Fagus grandifolia logs for commercial spalting applications," Journal of Wood Science, vol. 59, no. 4, pp. 351-357, 2013.

[13] S. C. Robinson, D. Tudor, H. Snider, and P. A. Cooper, "Stimulating growth and xylindein production of Chlorociboria aeruginascens in agar-based systems," AMB Express, vol. 2, no. 1, pp. 1-7, 2012.

[14] S. C. Robinson, E. Hinsch, G. Weber, and S. Freitas, "Method of extraction and resolubilisation of pigments from Chlorociboria aeruginosa and Scytalidium cuboideum, two prolific spalting fungi," Coloration Technology, vol. 130, no. 3, pp. 221-225, 2014.

[15] S. C. Robinson, E. Hinsch, G. Weber, K. Leipus, and D. Cerney, "Wood colorization through pressure treating: the potential of extracted pigments from spalting fungi as a replacement for woodworkers' aniline dyes," Materials, vol. 7, no. 8, pp. 54275437, 2014.

[16] G. Weber, H.-L. Chen, E. Hinsch, S. Freitas, and S. C. Robinson, "Pigments extracted from the wood-staining fungi Chlorociboria aeruginosa, Scytalidium cuboideum, and S. ganodermophthorum show potential for use as textile dyes," Coloration Technology. In press.

[17] S. C. Robinson, D. Tudor, W. R. Zhang, S. Ng, and P. A. Cooper, "Ability of three yellow pigment producing fungi to colour wood under controlled conditions," International Wood Products Journal, vol. 5, no. 2, pp. 103-107, 2014.

[18] S. C. Robinson, P. E. Laks, and E. J. Turnquist, "A method for digital color analysis of spalted wood using scion image software," Materials, vol. 2, no. 1, pp. 62-75, 2009.

[19] R. Bergman, "Drying and control of moisture content and dimensional changes. Wood handbook-wood as an engineering material," General Technical Report FPL-GTR-113, Department of Agriculture, Forest Service, Forest Products Laboratory, Madison, Wis, USA, 2010.

[20] R. B. Keey, T. A. G. Langrish, and J. C. F. Walker, Kiln-Drying of Lumber, Springer, Berlin, Germany, 2000.
[21] G. Job and F. Herrmann, "Chemical potential-a quantity in search of recognition," European Journal of Physics, vol. 27, no. 2, pp. 353-371, 2006.

[22] R. B. Hoadley, Identifying Wood: Accurate Results with Simple Tools, Taunton Press, Newtown, Conn, USA, 1990.

[23] J. Zheng and M. D. Fayer, "Solute-solvent complex kinetics and thermodynamics probed by $2 \mathrm{D}$-IR vibrational echo chemical exchange spectroscopy," Journal of Physical Chemistry B, vol.112, no. 33, pp. 10221-10227, 2008.

[24] W. J. Genck, "Make the most of antisolvent crystallization," Chemical Processing, 2010, http://www.chemicalprocessing .com/articles/2010/210/.

[25] W. J. Genck, "Better growth in batch crystallizers," Chemical Engineering, vol. 107, no. 8, pp. 90-95, 2000. 

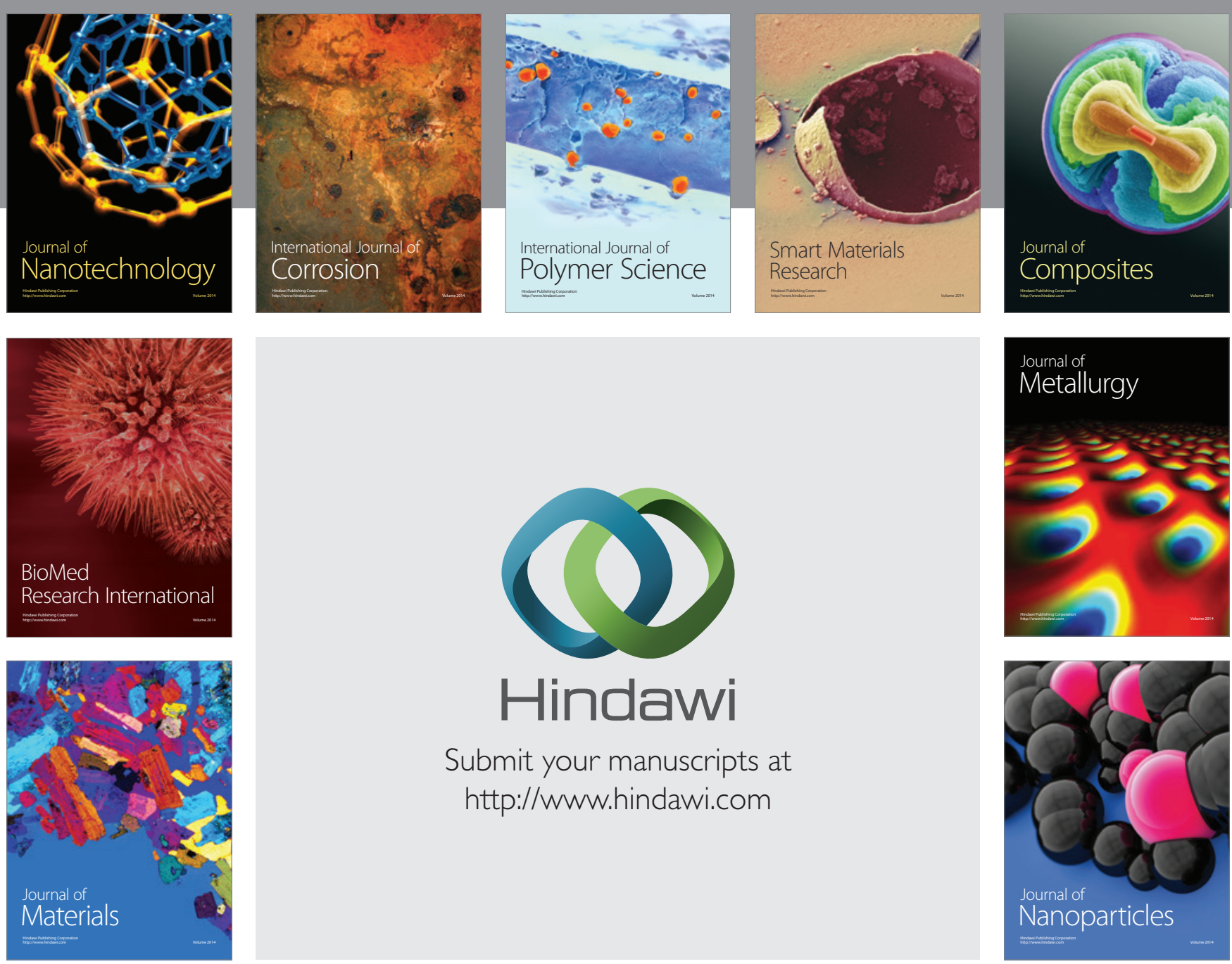

Submit your manuscripts at http://www.hindawi.com
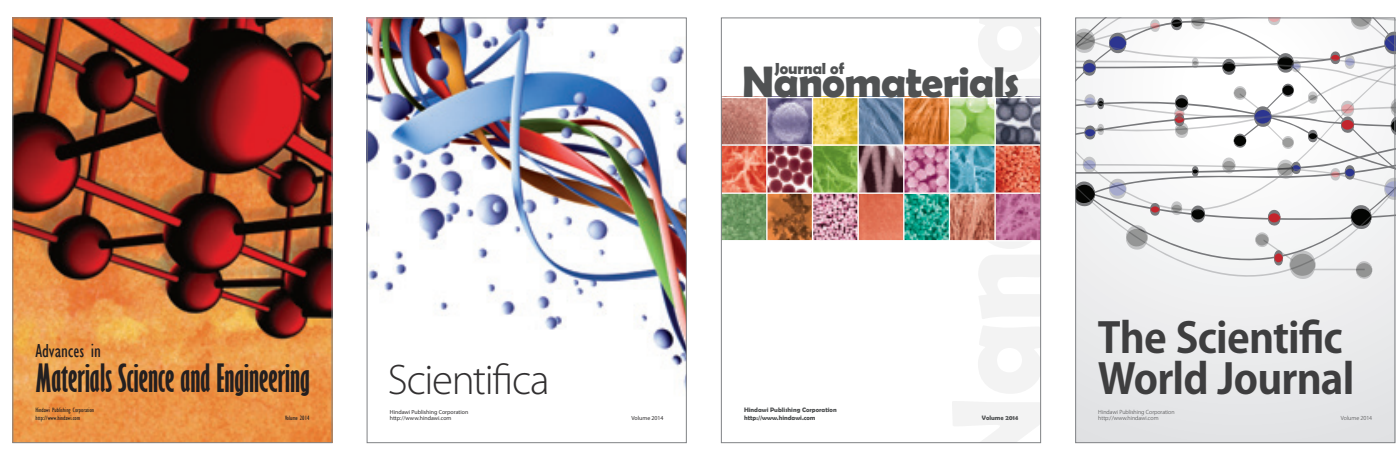

\section{The Scientific World Journal}
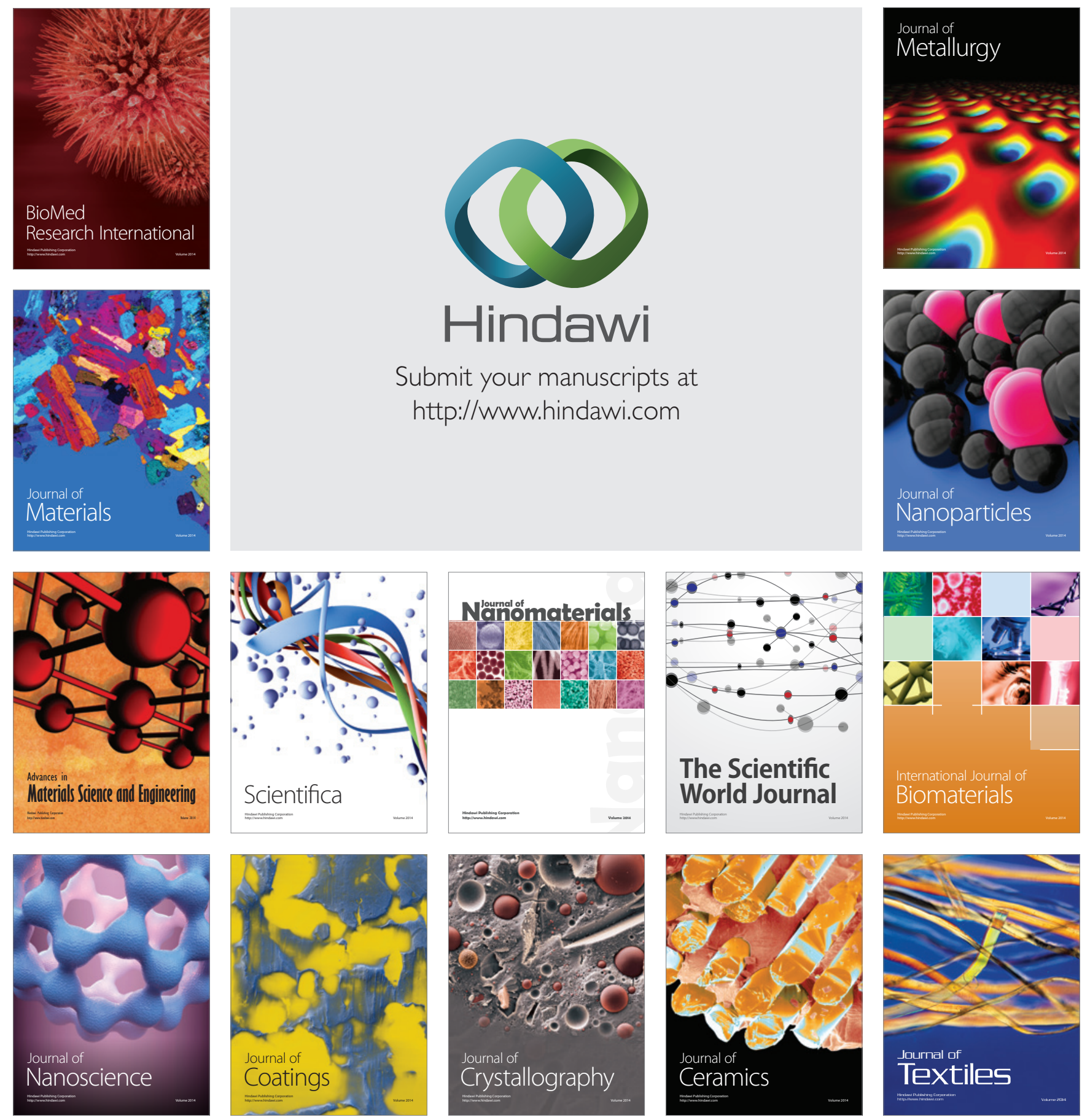